\title{
Infra-renal abdominal aortic calcification volume does not predict small abdominal aortic aneurysm growth
}

\author{
Kerolos Hendy a, Ronny Gunnarsson b, c, d, Oliver Cronin a , Jonathan Golledge a, e, * \\ ${ }^{a}$ Vascular Biology Unit, Queensland Research Centre for Peripheral Vascular Disease, College of Medicine and Dentistry, James Cook University, Townsville, \\ QLD, 4811, Australia \\ ${ }^{\mathrm{b}}$ Cairns Clinical School, Cairns Base Hospital, College of Medicine and Dentistry, James Cook University, Townsville, QLD, 4811, Australia \\ ${ }^{\mathrm{c}}$ Research and Development Unit, Primary Health Care and Dental Care Southern Älvsborg County Region Västra Götaland, Sweden \\ ${ }^{\mathrm{d}}$ Department of Public Health and Community Medicine Institute of Medicine, The Sahlgrenska Academy, University of Gothenburg, Sweden \\ e Department of Vascular and Endovascular Surgery, The Townsville Hospital, Townsville, QLD, 4811, Australia
}

\section{A R T I C L E I N F O}

\section{Article history:}

Received 2 April 2015

Received in revised form

10 July 2015

Accepted 14 July 2015

Available online 16 July 2015

\section{Keywords:}

Abdominal aortic aneurysm

Growth

Calcification

\begin{abstract}
A B S T R A C T
Background: Vascular calcification is a common finding in abdominal aortic aneurysms (AAA) however whether it predicts aneurysm expansion is controversial.

Objectives: 1) To establish a reproducible method of assessing AAA calcification using computed tomography (CT); 2) To investigate the association between AAA calcification and growth.

Method: Patients were identified from a prospectively maintained small AAA surveillance database. To be included patients required at least two CT scans a minimum of 6 months apart. All patients had a maximal AAA diameter of $\leq 55 \mathrm{~mm}$ on their initial scan. Infra-renal aortic calcification volume, total infra-renal aortic volume and maximal AAA diameter were measured. Reproducibility was assessed from repeat scans performed on 31 patients. AAA growth, estimated by volume change per year, was compared between patients with baseline infra-renal aortic calcification volumes $<$ and $\geq$ median.

Results: 95\% agreement limits (lower, upper) for intra and inter-observer error in measuring infra-renal aortic calcification volume were $0.68,97 \mathrm{~mm}^{3}$ and $-140,5.8 \mathrm{~mm}^{3}$, respectively. Concordance correlation coefficients for inter and intra-observer variability in measuring infra-renal aortic calcification volume were 0.99 and 0.99 , respectively. Patients with infra-renal aortic calcification volume $<$ median $(n=44)$ and $\geq$ median $(\mathrm{n}=44)$ had an infra-renal aortic volume increase of $6.0 \mathrm{~cm}^{3} / \mathrm{yr}$ and $7.8 \mathrm{~cm}^{3} / \mathrm{yr}$, respectively $(\mathrm{p}=0.66)$. Mean percentage infra-renal aortic volume increase/yr was found to be $4.2 \pm 6.4$ and $8.9 \pm 6.2$ for patients with and without diabetes, respectively $(\mathrm{p}=0.003)$.

Conclusion: Infra-renal aortic calcification volume can be assessed reproducibly from CT images. Infrarenal aortic calcification volume did not predict small AAA growth.
\end{abstract}

Crown Copyright $\odot 2015$ Published by Elsevier Ireland Ltd. All rights reserved.

\section{Introduction}

Abdominal aortic aneurysm (AAA) is an important cause of mortality in older adults. There are currently no established drug therapies to limit AAA growth and surgical intervention does not reduce mortality of patients with small AAAs [1-3]. As a result, most patients with small AAAs undergo imaging surveillance until AAA diameter is $\geq 50-55 \mathrm{~mm}(\mathrm{~mm})$. Despite such surveillance,

\footnotetext{
* Corresponding author. Vascular Biology Unit, Queensland Research Centre for Peripheral Vascular Disease, College of Medicine and Dentistry, James Cook University, Townsville, QLD, 4811, Australia.
}

E-mail address: jonathan.golledge@jcu.edu.au (J. Golledge).
$1-2 \%$ of small AAAs rupture per year [4,5]. Additional measures of selecting patients for intervention are needed [6].

AAA rupture represents a mechanical failure of the degenerated aortic wall, thus biomechanical considerations are important to understand this process and to improve our predictions of its occurrence [7]. Patient specific biomechanical profiling has been suggested as a potentially valuable tool in rupture risk assessment [8,9]. Additionally, peak wall stress (PWS) has been reported to be greater in ruptured and symptomatic AAAs compared to asymptomatic AAAs [10], and PWS has been reported to predict location of future rupture [9,11]. Furthermore, finite element analysis (FEA) studies have demonstrated that PWS is significantly greater in AAA regions with calcified plaque compared to regions with no 
calcification [12]. Abdominal aortic calcification (AAC) score has also been reported to be higher in symptomatic and ruptured AAAs compared to asymptomatic AAAs [13]. A recent study evaluating AAA tissue specimen has implicated AAA calcification in determining rupture risk [14]. However, a multi-detector computed tomography study suggested that calcified plaques are smaller in AAA compared to normal abdominal aortas [15]. Thus the role of calcification in AAA pathogenesis has not been clearly elucidated.

A previous study reported that greater AAA calcification was associated with slower AAA expansion. In that study calcification was assessed semi-quantitatively by ultrasound (US) and interobserver reproducibility was not reported. Furthermore analyses did not adjust for baseline AAA size [16]. A further small study which used computed tomography (CT) imaging to assess AAA calcification reported no association between AAA calcification volume and growth [17]. The nature of the relationship between infra-renal aortic calcification volume and small AAA growth is yet to be resolved. Studies investigating AAA calcification have employed variable methods of quantifying calcification and frequently not reported the assessment reproducibility [18].

The aims of the current study were:

1. To establish a valid and reproducible method of assessing infrarenal aortic calcification with CT;

2. To investigate the association between infra-renal aortic calcification volume and AAA growth rate.

\section{Methods}

\subsection{Patients and clinical definitions}

We performed a retrospective analysis of serial CT images of patients with small AAAs that were under surveillance at The Townsville Hospital (TTH) between June 2003 and November 2013. Such patients had previously consented to their medical information being confidentially stored in a database and used for research purposes. A low, negligible risk ethics application for this study was approved by TTH Human Research and Ethics Committee (HREC) (approval number 13/QTHS/125) and endorsed by James Cook University HREC.

To be included patients had to have undergone at least two CT scans a minimum of 6 months apart and images had to be available for retrospective analysis. All patients had a maximal axial AAA diameter of $\leq 55 \mathrm{~mm}$ on their initial scan. Repaired and mycotic AAAs were excluded. Patients with connective tissue diseases, such as Marfan's syndrome, were excluded.

Ischemic heart disease (IHD) was defined by a history of myocardial infarction, angina pectoris or coronary revascularisation. Hypertension and diabetes mellitus (DM) were defined by previous history or treatment for these conditions. Cigarette smoking classification was based on smoking history and defined as current smoker (smoked within the last month), ex-smoker (smoked previously but not in the last month) or never smoked.

\subsection{CT acquisition}

All CTs were performed at TTH using a 64 slice multi scanner (Philips, North Ryde, NSW), under a set acquisition protocol [19]. Abdominal aortic images were obtained in a single breath hold cycle in $3 \mathrm{~mm}$ slices at $3 \mathrm{~mm}$ intervals. $100 \mathrm{~mL}$ Ultravist 300 was delivered intravenously using an automated injection driver system. Image capture commenced when the locater set above the renal arteries detected that Hounsfield units at the centre of the aorta reached 130 .

\subsection{Assessment of infra-renal aortic calcification}

Original CT images were transferred onto a Philips MxView Visualisation Workstation software for detailed analysis. The region of interest (ROI) was the slice inferior to the origin of the lowest renal artery to the slice superior to the bifurcation of the abdominal aorta. Firstly, axial images throughout the ROI were scouted to carefully demarcate the outer boundary of the aortic wall. The outer aortic wall was traced free hand with the cursor under magnified images to improve accuracy and to exclude adjacent bowel tissue, vertebral body tissue and all non-aortic tissue from the ROI. Secondly, coronal images were also scouted to identify the upper and lower boundaries of the ROI as described above. Calcification volume was subsequently extracted from the ROI using a workstation tool based on predefined and validated thresholds of radio density for calcification [19] as illustrated in Fig. 1. Results were transcribed onto an excel spreadsheet for further analysis.

\subsection{Assessment of infra-renal aortic volume and AAA diameters}

Infra-renal aortic volume was assessed using methods similar to those used to measure infra-renal aortic calcification volume. After selecting the ROI and abdominal aortic boundary from axial slices, the total infra-renal aortic volume was estimated based on predefined and validated radio density set up on the Phillips workstation [19]. AAA orthogonal diameter was measured by first scouting axial images within the ROI to identify the abdominal aortic centre point in each axial slice. Subsequently, images perpendicular to the AAA centreline were analysed to measure the maximal orthogonal diameter including the outer wall and thrombus. AAA growth was estimated from two CT scans, at least six months apart, for all patients. Growth was calculated by normalising the respective volume and diameter changes relative to the time interval between CTs for each patient and reporting as changes per year.

\subsection{Reproducibility}

In order to determine the repeatability of calcification measurements we assessed the agreement of measurements between and within observers on 31 patients. This involved two final year medical students, each trained by an experienced doctor, measuring and recording the calcification volume from CTs whilst being blinded to each other's results. Measurements were then repeated at least $24 \mathrm{~h}$ later by one observer $(\mathrm{KH})$ to assess the intraobserver error.

\subsection{Statistical analysis}

Statistical analysis for reproducibility was performed according the methods outlined by Bland and colleagues [20]. Furthermore, concordance correlation coefficient were calculated according to Lin [21].

To test our hypothesis that patients with infra-renal aortic calcification volume below median would experience a greater AAA volume increase compared to those with calcification volume equal to or above median, patients were divided into two groups. Group 1 has infra-renal aortic calcification volume less than median and group 2 has an infra-renal aortic calcification volume greater than or equal to median.

The required sample size was calculated based on two assumptions. Firstly, mean AAA volume growth/year in patients with calcification volume < median was assumed to be $12 \mathrm{~cm}^{3} / \mathrm{yr}$, $\mathrm{SD}=6.5 \mathrm{~cm}^{3} / \mathrm{yr}$ based on results from a previous CT study [17]. Secondly we predicted that AAA growth rate would be $42 \%$ greater 


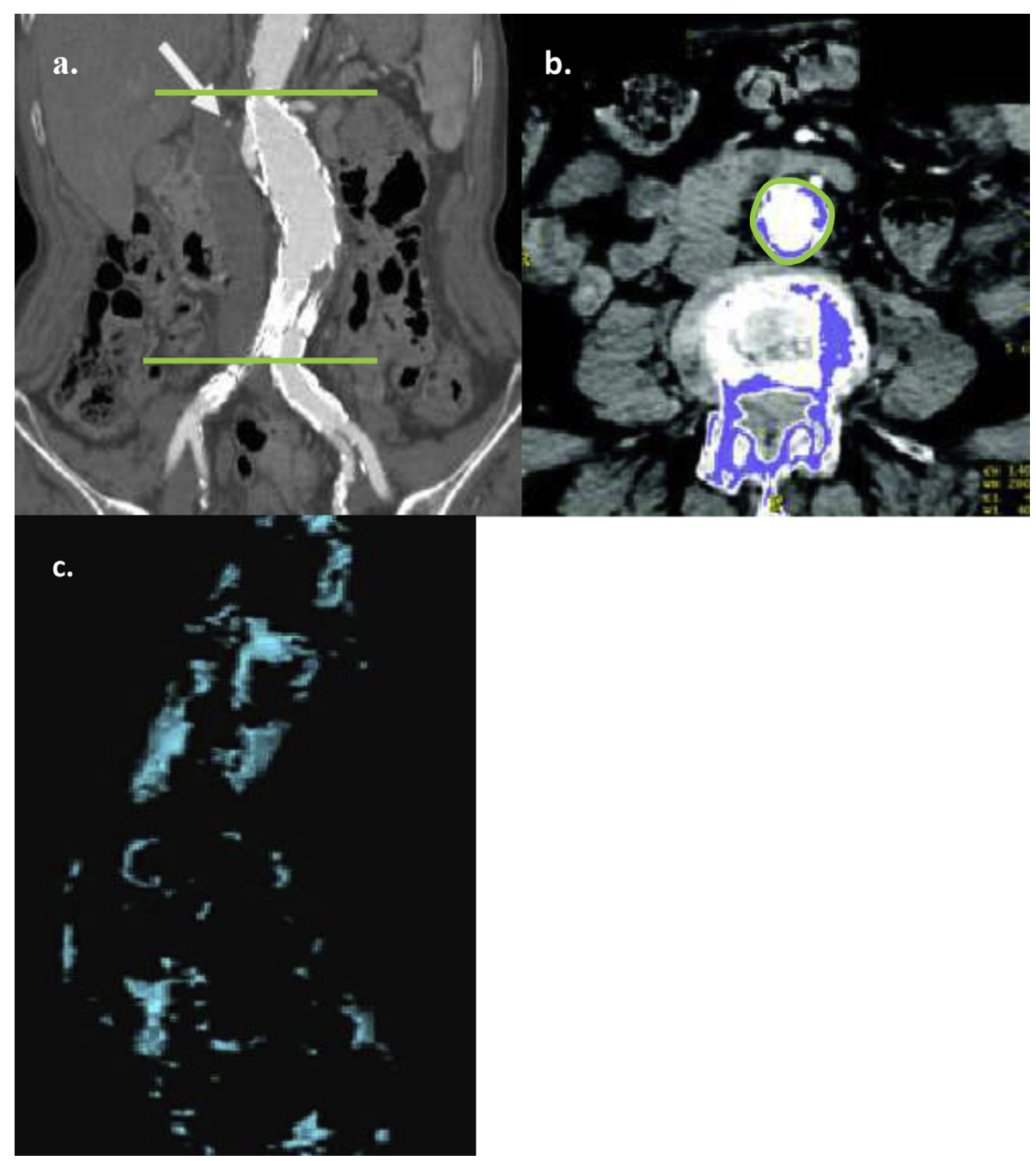

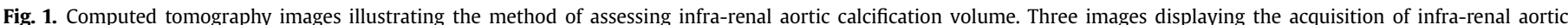

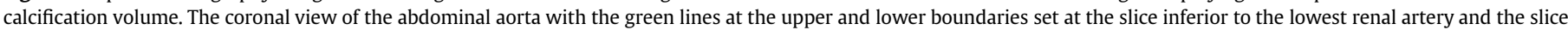

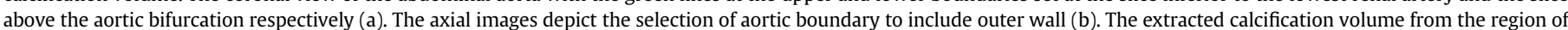

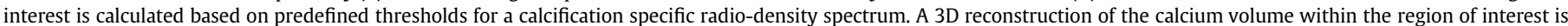
depicted in (c).

in patients with calcification volume $<$ median as suggested by results from a study by Lindholt and colleagues [16]. Using the Gpower 3.1.9.2 tool, (Two tailed t-test: difference between means $\alpha=0.05$, Power $=0.95$ ), 30 observations in each group were needed.

All results were transcribed onto an excel spreadsheet and later transferred to SPSS version 22 (IBM SPSS) for statistical analysis. Chi squared t-test and Mann-Whitney $U$ test were used to compare nominal and continuous variables, respectively, between groups. Medians and interquartile ranges were reported for variables which were not normally distributed and means and standard deviations were used to describe results for continuous variables following a normal distribution. Correlation analyses were performed between continuous variables where the dependant variable followed a normal distribution and reported as Pearson's correlation coefficient.

\section{Results}

Records of 173 patients who underwent serial CT imaging of their AAA between June 2003 and Nov 2013 were screened for inclusion into the study. A total of 88 patients met eligibility criteria and were included. Median time interval between the two CT scans assessed from each patient was 16 months, interquartile range 12-28 months. Thirty percent of the cohort were current smokers at recruitment and $74 \%$ were male. The median baseline infra-renal aortic calcification volume for the cohort was $1600 \mathrm{~mm}^{3}$, interquartile range of $830-2800 \mathrm{~mm}^{3}$. Median baseline infra-renal aortic volume was $91 \mathrm{~cm}^{3}$ with an interquartile range of $73-108 \mathrm{~cm}^{3}$. Mean baseline orthogonal diameter was $43.0 \mathrm{~mm}$, standard deviation was $5.5 \mathrm{~mm}$.

\subsection{Reproducibility}

31 subjects were involved in the reproducibility study. Interobserver and intra-observer 95\% limits of agreement for infrarenal aortic calcification volume were (lower, upper): -140 , $5.8 \mathrm{~mm}^{3}$ and $0.68,97 \mathrm{~mm}^{3}$, respectively. Ninety-five percent limits of agreement for infra-renal aortic volume were (lower, upper); $-4.5,0.027 \mathrm{~cm}^{3}$ and $-0.007,3.0 \mathrm{~cm}^{3}$ for inter and intraobserver error respectively. The concordance correlation coefficients (CCC) for inter-observer and intra-observer variability in 
Table 1

Reproducibility assessment.

\begin{tabular}{|c|c|c|}
\hline & $\begin{array}{l}\text { 95\% Limits of agreement } \\
\text { (lower, upper) }\end{array}$ & $\begin{array}{l}\text { Concordance correlation } \\
\text { coefficient }(95 \% \mathrm{CI})\end{array}$ \\
\hline \multicolumn{3}{|l|}{ Intra observer error } \\
\hline Infra-renal aortic volume $\left(\mathrm{cm}^{3}\right)$ & $-0.007,3.0$ & $0.99(0.99-1.0)$ \\
\hline Infra-renal aortic calcification volume $\left(\mathrm{mm}^{3}\right)$ & $0.68,97$ & $0.99(0.998-1.0)$ \\
\hline \multicolumn{3}{|l|}{ Inter observer error } \\
\hline Total Infra-renal aortic volume $\left(\mathrm{cm}^{3}\right)$ & $-4.5,0.027$ & $0.98(0.97-0.99)$ \\
\hline Infra-renal aortic calcification volume $\left(\mathrm{mm}^{3}\right)$ & $-140,5.8$ & $0.99(0.99-1.0)$ \\
\hline
\end{tabular}

CI: confidence interval.

Table 2

Clinical risk factors in patients with infra-renal aortic calcification volumes $<$ median and $\geq$ median.

\begin{tabular}{|c|c|c|c|c|}
\hline & Total cohort $(\mathrm{n}=88)$ & $<\operatorname{median}(\mathrm{n}=44)$ & $\geq \operatorname{median}(\mathrm{n}=44)$ & P-value \\
\hline Male gender & $65(74 \%)$ & $35(80 \%)$ & $30(68 \%)$ & 0.33 \\
\hline Current smoker & $26(30 \%)$ & $9(20 \%)$ & $17(39 \%)$ & 0.10 \\
\hline Diabetes mellitus & $24(27 \%)$ & $12(27 \%)$ & $12(27 \%)$ & 1.0 \\
\hline Hypertension & $65(74 \%)$ & $30(68 \%)$ & $35(80 \%)$ & 0.33 \\
\hline Ischemic heart disease & $46(52 \%)$ & $25(57 \%)$ & $21(48 \%)$ & 0.52 \\
\hline Calcium channel blocker & $22(25 \%)$ & $10(23 \%)$ & $12(27 \%)$ & 0.81 \\
\hline ARB & $17(19 \%)$ & $12(27 \%)$ & $5(11 \%)$ & 0.10 \\
\hline ACE inhibition & $35(40 \%)$ & $17(39 \%)$ & $18(41 \%)$ & 1.0 \\
\hline
\end{tabular}

ARB: Angiotensin II receptor blocker. ACE: Angiotensin converting enzyme.

measuring infra-renal aortic volume were 0.98 and 0.99 , respectively. In measuring infra-renal aortic calcification volume the CCCs were 0.99 and 0.99 for inter-observer and intra-observer variability, respectively (Table 1 ).

\subsection{Association of aortic calcification with AAA expansion}

None of baseline characteristics assessed were significantly different between the groups with $<$ median or $\geq$ median calcification volume (Table 2). Median infra-renal aortic volume increase in the group with $<$ median calcification was $6.0 \mathrm{~cm}^{3} / \mathrm{yr}$ compared to $7.8 \mathrm{~cm}^{3} / \mathrm{yr}$ in the group with $\geq$ median $(\mathrm{p}=0.66)$. Median AAA orthogonal diameter increase was $1.6 \mathrm{~mm} / \mathrm{yr}$ in the group with $<$ median calcification and $1.8 \mathrm{~mm} / \mathrm{yr}$ for the group with $\geq$ median $(\mathrm{p}=0.99)$. Actual and percentage AAA growth rates were similar in both groups (Table 3 ). Infra-renal aortic calcification volume had minimal correlation with percentage AAA volume change/yr (Pearson correlation $=0.10, \mathrm{p}=0.36$ ). Mean percentage AAA volume change/yr was $4.2 \pm 6.4 \%$ and $8.9 \pm 6.2 \%$ for patients with and without diabetes, respectively ( $\mathrm{p}=0.003$ ).

\section{Discussion}

The findings from this study suggest that infra-renal aortic calcification volume can be measured from CT with good inter and intra-observer reproducibility compared to other published studies on the subject $[17,22]$. Results from this cohort suggest that infrarenal aortic calcification volume is not significantly associated with small AAA growth rates. Findings from this cohort of patients are consistent with previous US and CT based studies in reporting that diabetes mellitus is associated with reduced AAA growth rates $[1,23,24]$.

Patients with diabetes are believed to have more marked vascular calcification and thus one possible explanation for reduced AAA expansion in these patients could be through more advanced aortic calcification [25]. Our findings however suggest that aortic calcification does not explain the reduced AAA expansion in patients with diabetes. The changes seen in the walls of aneurysmal aortas include inflammation and the activation of proteolytic pathways associated with loss of elastin and other structural proteins [26]. In contrast, diabetes is associated with increased synthesis and reduced degradation of extracellular matrix. The deposition of advanced glycosylation end products also renders vascular matrix resistant to proteolysis and inflammation in patients with diabetes [25]. It is likely these changes in the extracellular matrix are responsible for the reduced AAA expansion in patients with diabetes rather than any effects of aortic calcification.

Previous studies have alluded to the potential that AAA calcification may be a novel additional tool for AAA rupture risk assessment [13]. This has been supported by FEA findings of increased PWS in AAA regions with calcified plaque [9-11]. However our results did not suggest a significant association between infra-renal aortic calcification volume and AAA growth. This suggests that incorporating AAA calcification in rupture risk assessment is unlikely to improve accuracy of predicting rupture, although a larger long term prospective study would be needed to confirm this. Thus

Table 3

Comparison of AAA growth in patients with infra-renal aortic calcification volume $<$ median and $\geq$ median.

\begin{tabular}{|c|c|c|c|c|}
\hline & Total cohort $(\mathrm{n}=88)$ & $<$ Median $(\mathrm{n}=44)$ & $\geq$ Median $(\mathrm{n}=44)$ & P-value \\
\hline Age (years) & $72(66,77)$ & $71(65,76)$ & $73(68,78)$ & 0.13 \\
\hline Infra-renal aortic thrombus volume $\left(\mathrm{cm}^{3}\right)$ & $27(17,36)$ & $23(15,35)$ & $28(24,39)$ & 0.22 \\
\hline Volume change $\left(\mathrm{cm}^{3} /\right.$ year $)$ & $6.5(1.7,12)$ & $6.0(1.7,12)$ & $7.8(1.7,12)$ & 0.66 \\
\hline Orthogonal diameter change (mm/year) & $1.7(0.66,2.9)$ & $1.6(0.7,3.0)$ & $1.8(0.5,2.9)$ & 0.99 \\
\hline Volume change/year (\%) & $7.3(3.0,12)$ & $8.0(2.8,12)$ & $7.2(3.1,11)$ & 0.80 \\
\hline Orthogonal diameter change/year (\%) & $3.6(1.7,6.6)$ & $3.4(1.9,7.5)$ & $4.1(1.1,6.4)$ & 0.89 \\
\hline
\end{tabular}

$\mathrm{Cm}$ : centimetres. P-value is reported comparing group 1 and group 2. 
we currently have insufficient evidence to necessitate consideration of infra-renal aortic calcification volume when selecting patients for intervention. Additionally, this study contributes to the evidence refuting the theory that suggests heavily calcified AAAs grow slower than less calcified AAAs. This is important in avoiding misconceived alterations to AAA surveillance periods based on the degree of AAA calcification. Our current data suggests that calcified AAAs should have similar imaging surveillance as other AAAs. Moreover, our findings do not support the exploration of pharmacological manipulation of AAA calcification as a means to slow expansion of AAAs.

This study has a number of limitations. Firstly, only 88 patients were included in the current study. We based this on a sample size calculation which assumed a $42 \%$ difference in AAA growth rate between the two groups thus we were underpowered to examine smaller differences in AAA growth. We assessed AAA growth from $\mathrm{CT}$ scans performed at varying intervals. In order to adjust for these differences in time intervals we had to assume linear AAA growth which is not always the case although we found this to be present in a recent cohort [27]. Furthermore, the possibility of selection bias should be noted. Patients who undergo CT rather than US surveillance are commonly those with one or more of the following attributes: obesity, a particularly tortuous aorta, and those with a large baseline AAA diameter. On the other hand, patients presumably omitted from this cohort include those who may need CT imaging but are at increased risk of contrast induced nephropathy. Finally we only assessed aortic calcification volume. It is conceivable that other aspects of calcification like is distribution or thickness might have separate impact on the growth of small AAAs however we suspect this is unlikely given the lack of association of calcification volume.

In conclusion despite the established association of vascular calcification with increased incidence of cardiovascular events [28], infrarenal aortic calcification volume does not appear to be associated with small AAA growth. The finding of the current study is consistent with findings from a previous study by our group [17].

\section{Conflict of interest}

Authors declare no conflict of interest.

\section{Acknowledgements}

JG is supported by grants from the National Health and Medical Research Council [project grants 1063476, 1022752, 1021416, 1020955, 1003707; Centre of Research Excellence 1000967; fellowship 1019921], and The Office of Health and Medical Research, Queensland Government. JG holds a Practitioner Fellowships from the National Health and Medical Research Council, Australia (1019921). JG holds a Senior Clinical Research Fellowship from the Office of Health and Medical Research, Queensland.

\section{References}

[1] M.J. Sweeting, S.G. Thompson, L.C. Brown, J.T. Powell, collaborators RESCAN, Meta-analysis of individual patient data to examine factors affecting growth and rupture of small abdominal aortic aneurysms, Br. J. Surg. 99 (5) (2012) 655-665.

[2] D. Bergqvist, Pharmacological interventions to attenuate the expansion of abdominal aortic aneurysm (AAA) - a systematic review, Eur. J. Vasc. Endovasc. Surg. 41 (5) (2011) 663-667.

[3] J. Golledge, P.E. Norman, Current status of medical management for abdominal aortic aneurysm, Atherosclerosis 217 (1) (2011) 57-63.

[4] J.T. Powell, S.M. Gotensparre, M.J. Sweeting, L.C. Brown, F.G. Fowkes, S.G. Thompson, Rupture rates of small abdominal aortic aneurysms: a systematic review of the literature, Eur. J. Vasc. Endovasc. Surg. 41 (1) (2011) $2-10$.

[5] S.G. Thompson, L.C. Brown, M.J. Sweeting, M.J. Bown, L.G. Kim, M.J. Glover, et al., Systematic review and meta-analysis of the growth and rupture rates of small abdominal aortic aneurysms: implications for surveillance intervals and their cost-effectiveness, Health Technol. Assess. 17 (41) (2013) 1-118.

[6] M. Fillinger, Who should we operate on and how do we decide: predicting rupture and survival in patients with aortic aneurysm, Semin. Vasc. Surg. 20 (2) (2007) 121-127

[7] D.A. Vorp, J.P. Vande Geest, Biomechanical determinants of abdominal aortic aneurysm rupture, Arterioscler. Thromb. Vasc. Biol. 25 (8) (2005) 1558-1566.

[8] A.H. Malkawi, R.J. Hinchliffe, Y. Xu, P.J. Holt, I.M. Loftus, M.M. Thompson, Patient-specific biomechanical profiling in abdominal aortic aneurysm development and rupture, J. Vasc. Surg. 52 (2) (2010) 480-488.

[9] T.C. Gasser, A. Nchimi, J. Swedenborg, J. Roy, N. Sakalihasan, D. Bockler, et al., A novel strategy to translate the biomechanical rupture risk of abdominal aortic aneurysms to their equivalent diameter risk: method and retrospective validation, Eur. J. Vasc. Endovasc. Surg. 47 (3) (2014) 288-295.

[10] S. Khosla, D.R. Morris, J.V. Moxon, P.J. Walker, T.C. Gasser, J. Golledge, Metaanalysis of peak wall stress in ruptured, symptomatic and intact abdominal aortic aneurysms, Br. J. Surg. 101 (11) (2014) 1350-1357.

[11] B.J. Doyle, T.M. McGloughlin, K. Miller, J.T. Powell, P.E. Norman, Regions of high wall stress can predict the future location of rupture of abdominal aortic aneurysm, Cardiovasc Interv. Radiol. 37 (3) (2014) 815-818.

[12] P. Erhart, C. Grond-Ginsbach, M. Hakimi, F. Lasitschka, S. Dihlmann, D. Bockler, et al., Finite element analysis of abdominal aortic aneurysms: predicted rupture risk correlates with aortic wall histology in individual patients, J. Endovasc. Ther. 21 (4) (2014) 556-564.

[13] R.V. Buijs, T.P. Willems, R.A. Tio, H.H. Boersma, I.F. Tielliu, R.H. Slart, et al., Calcification as a risk factor for rupture of abdominal aortic aneurysm, Eur. J. Vasc. Endovasc. Surg. 46 (5) (2013) 542-548.

[14] S.A. O'Leary, J.J. Mulvihill, H.E. Barrett, E.G. Kavanagh, M.T. Walsh, T.M. McGloughlin, et al., Determining the influence of calcification on the failure properties of abdominal aortic aneurysm (AAA) tissue, J. Mech. Behav. Biomed. Mater. 42 (2015) 154-167.

[15] C. Heilmaier, A. Koester, T. Moysidis, D. Weishaupt, K. Kroger, Abdominal aortic calcification and its distribution in normal-sized and aneurysmatic abdominal aortas, Vasa 43 (2) (2014) 132-140.

[16] J.S. Lindholt, Aneurysmal wall calcification predicts natural history of small abdominal aortic aneurysms, Atherosclerosis 197 (2) (2008) 673-678.

[17] A. Parr, S. McLaughlin, M. McLaughlin, J.G. McHir, Aortic calcification and abdominal aortic aneurysm expansion, Atherosclerosis 202 (2) (2009) 350.

[18] D.J. Bowden, S.R. Aitken, I.B. Wilkinson, A.K. Dixon, Interobserver variability in the measurement of abdominal aortic calcification using unenhanced $\mathrm{CT}$, Br. J. Radiol. 82 (973) (2009) 69-72.

[19] R.W. Jayalath, P. Jackson, J. Golledge, Quantification of abdominal aortic calcification on CT, Arterioscler. Thromb. Vasc. Biol. 26 (2) (2006) 429-430.

[20] J. Martin Bland, D.G. Altman, Statistical methods for assessing agreement between two methods of clinical measurement, Lancet 327 (8476) (1986) 307-310.

[21] K. Lin, A note on the concordance correlation coefficient, Biometrics 56 (2000) 324-325.

[22] A. Parr, C. Jayaratne, P. Buttner, J. Golledge, Comparison of volume and diameter measurement in assessing small abdominal aortic aneurysm expansion examined using computed tomographic angiography, Eur. J. Radiol. 79 (1) (2011) 42-47.

[23] J.T. Powell, M.J. Sweeting, L.C. Brown, S.M. Gotensparre, F.G. Fowkes, S.G. Thompson, Systematic review and meta-analysis of growth rates of small abdominal aortic aneurysms, Br. J. Surg. 98 (5) (2011) 609-618.

[24] K. Hendy, R. Gunnarson, J. Golledge, Growth rates of small abdominal aortic aneurysms assessed by computerised tomography-a systematic literature review, Atherosclerosis 235 (1) (2014) 182-188.

[25] J.C. Kovacic, V. Fuster, Vascular calcification, diabetes, and cardiovascular disease: connecting the dots, JACC Cardiovasc Imaging 5 (4) (2012) 367-369.

[26] J. Golledge, M. Karan, C.S. Moran, J. Muller, P. Clancy, A.E. Dear, et al., Reduced expansion rate of abdominal aortic aneurysms in patients with diabetes may be related to aberrant monocyte-matrix interactions, Eur. Heart J. 29 (5) (2008) 665-672.

[27] E.A. Sherer, R.R. Bies, P. Clancy, P.E. Norman, J. Golledge, Growth of screendetected abdominal aortic aneurysms in men: a bayesian analysis, CPT Pharmacometrics Syst. Pharmacol. 1 (2012) e12.

[28] F. Bastos Goncalves, M.T. Voute, S.E. Hoeks, M.B. Chonchol, E.E. Boersma R.J. Stolker, et al., Calcification of the abdominal aorta as an independent predictor of cardiovascular events: a meta-analysis, Heart 98 (13) (2012) 988-994. 